University of Louisville

ThinkIR: The University of Louisville's Institutional Repository

Electronic Theses and Dissertations

$5-2021$

\title{
Image Analysis of Charged Bimodal Colloidal Systems in Microgravity.
}

Adam J. Cecil

University of Louisville

Follow this and additional works at: https://ir.library.louisville.edu/etd

Part of the Complex Fluids Commons, Computer-Aided Engineering and Design Commons, Numerical Analysis and Scientific Computing Commons, Physical Processes Commons, and the Transport

Phenomena Commons

\section{Recommended Citation}

Cecil, Adam J., "Image Analysis of Charged Bimodal Colloidal Systems in Microgravity." (2021). Electronic Theses and Dissertations. Paper 3437.

https://doi.org/10.18297/etd/3437

This Master's Thesis is brought to you for free and open access by ThinkIR: The University of Louisville's Institutional Repository. It has been accepted for inclusion in Electronic Theses and Dissertations by an authorized administrator of ThinkIR: The University of Louisville's Institutional Repository. This title appears here courtesy of the author, who has retained all other copyrights. For more information, please contact thinkir@louisville.edu. 


\title{
IMAGE ANALYSIS OF CHARGED BIMODAL COLLOIDAL SYSTEMS IN
} MICROGRAVITY

By

Adam Josiah Cecil

B.S. University of Louisville, 2020

\author{
A Thesis \\ Submitted to the faculty of the \\ University of Louisville \\ J.B. Speed School of Engineering \\ as Partial Fulfillment for the Requirements \\ for the Degree of \\ Master of Engineering \\ In Chemical Engineering \\ Department of Chemical Engineering
}

April 2021 


\section{TABLE OF CONTENTS}

ACKNOWLEDGEMENTS

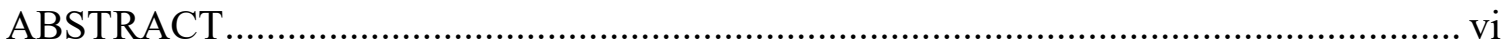

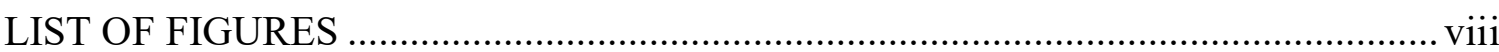

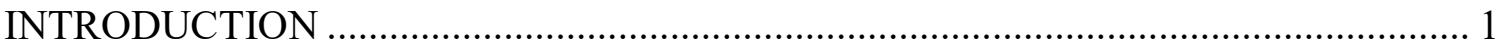

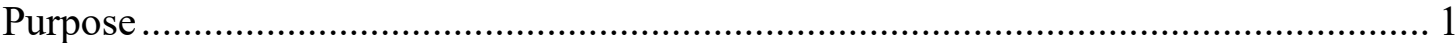

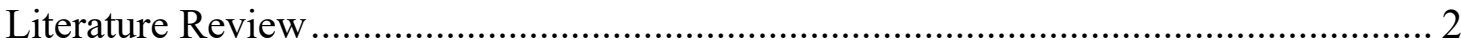

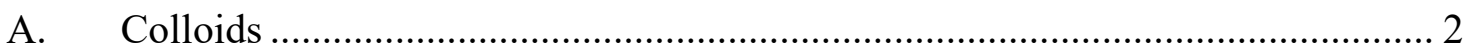

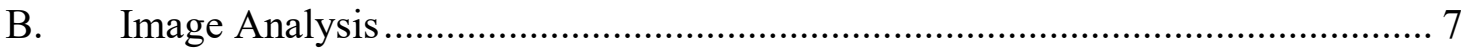

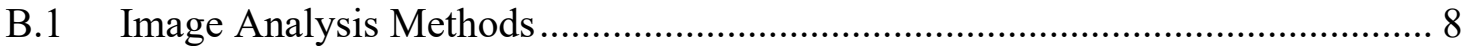

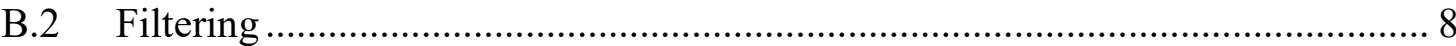

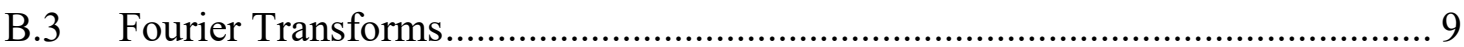

B.4 Image Analysis in Colloidal Science ............................................................ 11

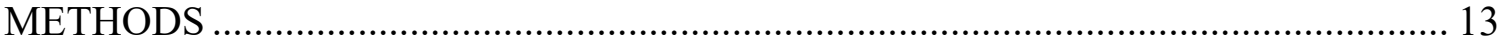

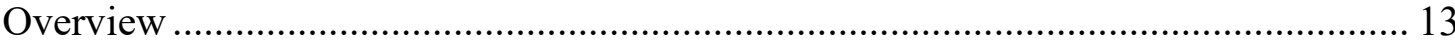

Silsesquioxane Microparticle Synthesis............................................................... 13

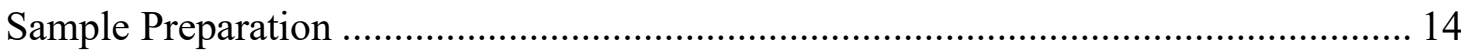

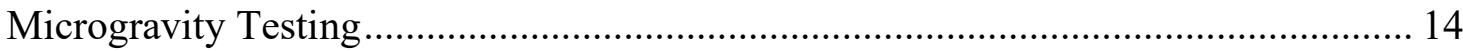

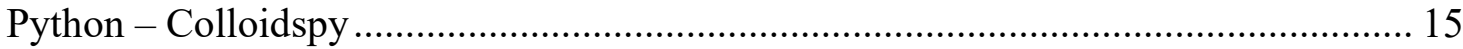

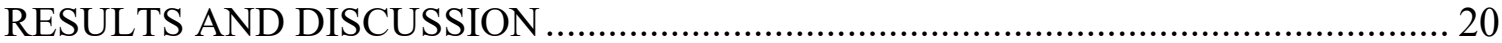


Cluster Size Analysis

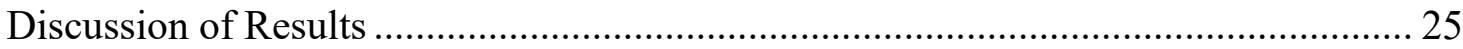

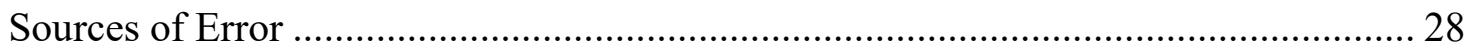

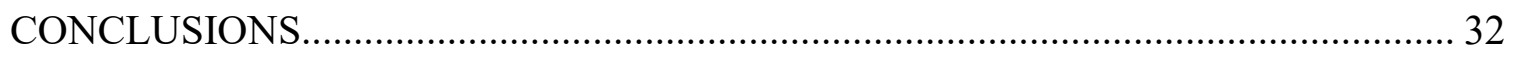

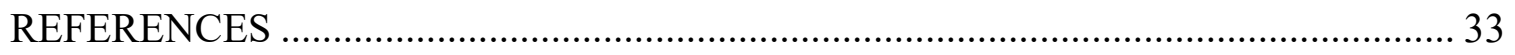




\title{
IMAGE ANALYSIS OF CHARGED BIMODAL COLLOIDAL SYSTEMS IN MICROGRAVITY
}

\author{
Submitted by:
}

Adam Josiah Cecil

A Thesis Approved on:

$4 / 12 / 2021$

By the following Reading and Examination Committee:

Dr. Gerold Willing, Thesis Chair

Dr. Stuart Williams, Committee Member

Dr. Vance Jaeger, Committee Member 


\section{ACKNOWLEDGEMENTS}

I would like to thank Dr. Willing and Dr. Williams for taking me onto the ACE-H2 team and allowing me to work with them on their research endeavors. Their continual support enabled me to learn and build upon the work of earlier researchers. I would also like to thank my family and my peers for supporting me throughout this journey. Specifically, I would like to thank my mother, Jacob Miller, and Peter Martin for many illuminating discussions.

In addition, I would like to thank the Stack Overflow community for their contributions to the many difficult programming challenges encountered in the development of this thesis. Finally, I would like to thank NASA and ZIN Technologies for their work in designing and executing the experiments studied in this thesis. 


\begin{abstract}
Colloids are suspensions of two or more phases and have been topics of research for advanced, tunable materials for decades. Stabilization of colloids is typically attributed to thermodynamic mechanisms; however, recent studies have identified transport or entropic mechanisms that can potentially stabilize a thermodynamically unstable colloidal system. In this study, suspensions of silsesquioxane microparticles and zirconia nanoparticles were dispersed in a nitric acid solution and allowed to aggregate for 8-12 days in microgravity aboard the International Space Station. The suspensions were subsequently imaged periodically at $2.5 x$ magnification. Due to the inadequacy of existing image analysis programs, the python package "Colloidspy" was developed to process these images and extract cluster boundaries. Trends of cluster area over time revealed two distinct behaviors. Some samples showed very low cluster growth rates and high cluster areas compared to microparticle size, while others showed an initial rapid growth phase, slowing to the low cluster growth rates of the other samples. The presence of this characteristic nonlinear phase coupled with the striking similarity in final growth rates of all samples suggests that the undocumented time between sample mixing and start of imaging may have been large enough that there was nonlinear growth in all samples, but only the final slow growth phase was captured in many of the samples. This type of behavior is not consistent with the linear agglomeration predictions of existing theory based on thermodynamic stabilization, but could be explained by the lack of buoyancy forces in microgravity allowing the space between clusters to increase as small clusters are incorporated into larger agglomerations. Further agglomeration could have been dominated by transport limitations even through the solution is still thermodynamically
\end{abstract}


unstable. Further study into the role of transport mechanisms in dilute colloidal systems in microgravity should be conducted to shed further light on this unusual behavior. 


\section{LIST OF FIGURES}

Figure 1: Basic silsesquioxane unit structure.

Figure 2: Left - a characteristic sample ROI; Right - the binarized version of the same ROI, where the white portion indicates a cluster. .15

Figure 3: Left - the binary image of an example ROI before cleaning. Right - the same binary image after cleaning.. .16

Figure 4: Left - an example ROI; Right - large cluster resulting from contour detection without use of the watershed algorithm... .17

Figure 5: Left - an example ROI; Right - clusters detected by the watershed algorithm are outlined in red, where the white portion is the cluster. .18

Figure 6: Left - average areas from Well 7, with $0.01 \% \mathrm{ZrO}_{2} ;$ Middle - average areas from Well 15, 0.1\% ZrO2; Right - average areas from Well $12-0.055 \% \mathrm{ZrO}_{2}$. 19

Figure 7: Left - total particle count from Well 7, with $0.01 \% \mathrm{ZrO}_{2}$; Middle - total particle count from Well 15, 0.1\% $\mathrm{ZrO}_{2}$; Right - total particle count from Well $12-0.055 \%$ $\mathrm{ZrO}_{2}$ .20

Figure 8: Spread of total particle count over all times for each well 21 Figure 9: Average cluster area over agglomeration time of all wells with $0.01 \% \mathrm{ZrO}_{2}$ nanoparticles. .22 Figure 10: Average cluster area over agglomeration time for all wells with $0.055 \% \mathrm{ZrO}_{2}$ nanoparticles. .23

Figure 11: Average cluster area over agglomeration time for all wells with $0.1 \% \mathrm{ZrO}_{2}$ nanoparticles. .24

Figure 12: Projected agglomerate area over number of particles. .30 


\section{INTRODUCTION}

\section{Purpose}

The characterization of colloidal suspensions is an essential component of colloid science and can be done in a variety of ways. In most cases scientists have direct, physical access to the suspensions they are studying, and can easily carry out tests such as TEM, XRD, static and dynamic light scattering, particle tracking, sedimentation velocity tests, and others. ${ }^{1-3}$ In this study, a colloidal suspension with a bimodal particle size and charge distribution was mixed and allowed to agglomerate in microgravity aboard the International Space Station (ISS). Because of a variety of factors - astronaut time, inability to receive the agglomerated samples back from the ISS, spatial and equipment restrictions - only stacks of $2.5 \mathrm{X}$ magnification images were obtained to study novel agglomeration behavior in the absence of gravity. Most traditional colloid analysis techniques could not be applied to this dataset, and existing image analysis programs failed to return consistently accurate results, so a new analysis pipeline was designed to extract meaningful data from these images. For this thesis, "Colloidspy," 4 a python package was developed to analyze these colloid images, and is presented and applied to images from these colloid systems in microgravity. Qualitative conclusions were developed from the dataset to direct and hone future microgravity colloidal agglomeration experiments and provide inspiration for new, more robust analysis techniques requiring only limited access to specialized equipment. 


\section{Literature Review}

\section{A. Colloids}

Colloids are heterogeneous suspensions of at least two distinct phases: a primary continuous phase, and a secondary dispersed phase. The phases can be solid, liquid, or gas, and each combination of phases forms a distinct subset of colloidal suspensions. For instance, a liquid-gas suspension is a foam, whereas a gas-liquid suspension is an aerosol. Common examples of other colloids include mayonnaise (liquid-liquid), gelatin (liquidsolid), and cheese (solid-liquid). Colloids are ubiquitous in modern-day materials, and advanced colloidal systems are being developed into new classes of highly tunable, functionalizable, self-assembling soft matter with applications ranging from biomatter to third-generation solar energy technology. ${ }^{5-7}$

Colloids were first acknowledged and studied by Francesco Selmi in the mid1800 s. $^{8}$ Selmi observed unusually low diffusion rates in certain solutions, later coined "colloids." These colloids were found to have large particle sizes compared to atoms, causing unexpected physical properties. In related work, Robert Brown observed continual movement of pollen grains in static water; this phenomenon was later named Brownian motion, and its first quantitative theory was developed in 1905 by Albert Einstein and Marian Smoluchowski. ${ }^{9}$

Early colloid theories built upon the work of Selmi, Einstein, Smoluchowski, and

others. One of the most groundbreaking models was developed by Asakura and Oosawa ${ }^{10}$ in 1954-1958, which changed the way interparticle forces were evaluated within colloidal systems. Asakura and Oosawa (A-O) suggested that van der Waals' and electrostatic forces were not the only major forces acting upon the particles, but that small spaces 
between particles experienced a type of osmotic pressure, causing an attractive depletion interaction between particles. To illustrate this, they modeled the suspensions as flat plates in suspension with hard sphere macromolecules - analogous to a suspension of particles significantly larger than the dispersed macromolecules. Two flat plates close enough to forcibly exclude the presence of macromolecules between them would experience osmotic pressure pushing the plates together due to the entropic tendency for the macromolecules to remove the concentration difference between the bulk and the space between the plates. They called this force the "depletion interaction," and extended this model to solutions with chain macromolecules, dissymmetrical macromolecules, and charged macromolecules to form a fairly comprehensive theory of colloidal aggregation for the time.

Thirty years later, Walz and Sharma further developed A-O theory on depletion interactions, this time using a force balance and second-order virial expansions to account for concentration effects and long-range electrostatic interactions between charged spheres $(5 \mu \mathrm{m})$ and charged macromolecules $(5 \mathrm{~nm})$ in solution. ${ }^{11}$ In the case of hard sphere-hard wall interactions, A-O's model was obtained. When the effects of higher macromolecule concentrations were included, they found repulsion interactions at large separations between spheres, unlike the attractive-only predictions of A-O. Upon addition of longrange electrostatic repulsion for macromolecule-macromolecule and spheremacromolecule interactions, all of the present depletion interactions were drastically amplified both in range and in magnitude. When considering both concentration effects and electrostatic repulsion at high concentrations ( $1 \mathrm{vol} \%$ macromolecules), they found attractive depletion interactions at close range, but repulsive long-range $(>50 \mathrm{~nm})$ depletion interactions. Of particular interest for this study is that at macromolecule concentrations 
below $0.5 \mathrm{vol} \%$, only attractive interactions were found over all range scales, becoming increasingly weaker with lower concentration. Even if the solution is electrostatically stabilized, the depletion attractions at lower concentrations could still cause flocculation, whereas high concentrations would enhance stability.

To further study the effects of concentration and polydispersity on colloidal solutions, Chu, Nikolov, and Wasan applied numerical solutions to the Ornstein-Zernike integral equation with the Percus-Yevick (PY) closure to colloidal systems of varying small-particle concentration and polydispersity. ${ }^{12}$ Their results showed a potential well near the large particles (distances less than the diameter of the small particles) corresponding to the A-O depletion region. However, contrary to A-O theory, there was a repulsive potential peak immediately after this region, and damped oscillatory behavior continuing as distance increases. Increasing small particle concentration strongly increased the depth of the potential well and moderately increased the height of the repulsive peak - the "energy barrier." Increasing size ratio weakly increased the depth of the potential well but strongly increased the height of the energy barrier. The fundamental process behind this behavior is that small particles form a structured layer around the larger particle in the attractive region. Energy is required to break this structure, blocking large particles from approaching each other. If the height of this barrier is significantly larger than $\mathrm{kT}$, there is not enough thermal energy in the colloidal solution to break this barrier, so the solution remains stable. However, at barrier heights near kT, large particles have enough thermal energy to occasionally break through the barrier, fall into the depletion potential well, and are trapped by the same energy barrier, causing long-term agglomeration. The effect of polydispersity follows logically: increasing polydispersity 
decreases the structural stability of the smaller-particle layer which decreases the energy barrier but increases the range of interaction because the interactions are less shielded.

In addition to thermodynamic stabilization mechanisms, teams of researchers at the University of Washington in 1988-1991 discovered that binary colloidal suspensions, both purely bimodal particles and particle-polymer solutions, could be stabilized through kinetic/transport mechanisms. ${ }^{13}, 14$ Using Monte Carlo simulations, analytical cluster variation calculations, and experiments, the researchers found that an initially stable suspension of one type of particle could be destabilized by increasing the concentration of either the polymer or the other particle type past a certain lower concentration. Upon increasing polymer or second particle concentration, the suspensions exhibited a peak flocculation rate, and beyond an upper concentration bound, restabilized. The cause of the restabilization was found not to be a thermodynamic stabilization, but simply the restriction of movement because of the high concentration of the secondary phase. An interesting consequence of the slowing of particle movement is long-term flocculation after the initial restabilization, suggesting that the true stable state of the system is the flocculated state, and the restabilization is only metastable.

Following a similar idea, in 2016 a group of researchers took an entropic approach to understanding the stabilization of binary charged colloidal systems. ${ }^{15}$ Traditional techniques generally focus on electrostatic or steric stabilization mechanisms. In contrast, their study examines the role of mixing entropy on colloidal stability, confirming that larger entropy of mixing results in more negative free energy, ultimately causing stabilization of the colloidal suspension, while phase separation is driven by positive free energies. While this idea of free energy driven stabilization is not new, they proposed that "attractive and 
unstable particles" ${ }^{15}$ can ultimately result in a stable suspension as long as the free energy of the final suspensions is negative.

Of interest to the series of experiments from which this thesis is derived, a groundbreaking study performed by Tohver, Chan, Sakurada, and Lewis in 2001 discovered unique assembly behavior in a special case of bimodal colloidal systems called nanoparticle haloing. ${ }^{16}$ Analogous to Chu et al.'s discovery of small-particle structures forming around larger colloidal particles, Tohver et al. found that highly charged nanoparticles form halos around negligibly charged microparticles. At nanoparticle concentrations within a narrow window, the solutions stabilized into a homogeneous fluid phase which settled into close-packed crystalline sediment after several days. However, at concentrations both above and below this concentration window, the suspension destabilized into colloidal gels. Later studies ${ }^{17,18}$ have confirmed this nanoparticle haloing theory, and also found that weak attraction between colloidal and nanoparticle species increases the concentration of nanoparticle halos immediately around the colloidal species. Increases in ionic strength of the suspension narrowed the stability region until nonexistent. ${ }^{19}$

Similar research on microparticle-nanoparticle suspensions was performed in 2018 by researchers from NDSU who performed computational simulations studying the stability of highly charged colloidal suspensions upon the addition of like-charged nanoparticles. ${ }^{20}$ Consistent with the theoretical framework, their simulations showed that addition of like-charged nanoparticles significantly weakened correlations between colloidal particles, while colloid-nanoparticle correlations increased with increasing charge, inducing the melting of colloidal crystals. 
Further research into dilute bimodal colloidal systems similar to the ones observed in the current study is scarce. Much of the recent research focuses on colloids within the protein-limit, where the dispersed phase is much smaller than the stabilizing phase (usually proteins), or nanoparticle haloing systems. Although the aim of the series of experiments from which this thesis is derived is to study nanoparticle haloing behavior in microgravity, the particular experiment studied in this thesis is not a true nanoparticle haloing system, as the microparticles are charged. Relevant computational studies report structure factors and $2^{\text {nd }}$-order virial coefficients, data for which are not available in this study, and should be pursued in future study.

\section{B. Image Analysis}

Digital Image analysis is the use of computational techniques to characterize objects or patterns in images ${ }^{21}$, making it extremely useful for automatically extracting experimental data from images of colloidal systems with relatively predictable features. Because digital images are simply arrays of numbers, mathematical techniques can be used to isolate specific characteristics to either extract or remove. These techniques include image thresholding, filtering, Fourier analysis, and more complicated techniques such as edge detection, depending on the type of characterization needed. For colloidal images, thresholding and filtering are useful for isolating particles from the background, and edge detection is useful for characterizing particle edges to extract metrics such as particle position, area, circularity, and perimeter. Fourier analysis can also be used for extracting information on the structure and regularity of particles and agglomerations. 


\section{B.1 Image Analysis Methods}

While binarization of images technically results in information loss, optimal binarization preserves only the features of interest while removing the background information. The least computationally intensive method for binarization is a global cutoff threshold, which sets all pixels greater than a given threshold value equal to True (or 255, for an 8-bit image), and all pixels less than that value to False (or 0). The most common type of global thresholding is Otsu thresholding. ${ }^{22}$ Otsu thresholding makes use of the tendency that the distribution of pixel values in many images are somewhat bimodal: the background is usually darker, the foreground lighter. The Otsu method calculates the pixel value in between these peaks that minimizes the difference between the spread of pixel values in the background vs. the foreground. This method works well with images of even lighting but struggles with lighting gradients.

A more computationally intensive but robust thresholding method is local, or adaptive thresholding, which evaluates the Boolean of pixels based on neighboring pixels. ${ }^{23}$ This method is useful because it is not as affected by lighting gradients, and with well-set parameters, can preserve more detail than most other thresholding methods. Several algorithms exist to perform local thresholding such as assigning pixel values based on the mean or median of neighboring pixels, or, the most common, using a Gaussian filter. ${ }^{24}$ Many other thresholding methods exist, comprehensively explored by Sankur in the Journal of Electronic Imaging, but are not relevant to this study. ${ }^{25}$

\section{B.2 Filtering}

Another aspect of image processing is filtering. Similar to how signal filters remove either high or low frequencies over time, image filters remove high or low 
frequency information over the spatial domain using convolution. ${ }^{26}$ A smooth lighting gradient across an image is a common example of low frequency information, whereas fine details like sharp edges are high frequency. Two of the most common filters are the Gaussian filter and the Sobel filter. The Gaussian filter is a type of low pass filter used to blur an image. Although detail is lost by blurring the image, random noise is also removed which can aid the computer in performing operations such as local thresholding with greater accuracy. ${ }^{27,28}$ Sobel filtering effectively removes low frequency information to isolate edges. This filter is essential for edge-detection, and is used extensively in computer vision. ${ }^{29}$

\section{B.3 Fourier Transforms}

The 2-dimensional Fourier Transform is another image analysis method that converts pixels in an image to their corresponding frequency space. This enables the extraction of information on length scales present in images and allows computationally simply convolution. Fourier analysis, named after Joseph Fourier, is used to express a function such as a signal or an image as a combination of sine and cosine waves. ${ }^{30}$ The formal definition of the Fourier transform is as follows:

$$
F(k)=\int_{-\infty}^{\infty} f(x) e^{-2 \pi i k x} d x
$$

where $F(k)$ is the Fourier transform of $f(x)$, and $\mathrm{k}$ is the wavenumber. ${ }^{31}$

For images, Fourier transforms take an image (spatial domain) and decompose it into its sine and cosine components, expressed as a frequency (or wavenumber) domain version of the image: 


$$
F(k, l)=\sum_{i=0}^{N-1} \sum_{j=0}^{N-1} f(i, j) e^{-i 2 \pi\left(\frac{k i}{N}+\frac{l j}{N}\right)}
$$

where $F(k, l)$ is the 2D dimensional Fourier transform of the image $f(i, j)$, which has $\mathrm{N} \times \mathrm{N}$ dimensions. $^{32}$

Each pixel in the Fourier transform of an image corresponds to a specific frequency contained in the original image, where the center pixels are the lowest frequency, moving to higher frequencies further out from the center. Blocking out a region of the Fourier transform and taking the inverse effectively applies a filter to the original image and can be used for a simple background subtraction if the centermost pixels are blocked in the Fourier transform. ${ }^{32}$ One important property of Fourier transforms is that addition in Fourier-space is the same as convolution in spatial or time domains. ${ }^{31}$ This property is central to image processing, including the efficient application of filters and other types of image characterizations that use convolution. ${ }^{26}$

Because Fourier transforms extract the frequency of length scales in images, they are useful for colloidal science to estimate structure factor. Structure factor is a measure of how a material scatters incident light and is directly related to the characteristic length between particles. Formally, the definition of structure factor is as follows: ${ }^{33}$

$$
S(q)=\frac{1}{N} \sum_{j}^{N} \sum_{k}^{N}\left\langle e^{i q\left(r_{j}-r_{k}\right)}\right\rangle
$$

where $q$ is the scattering vector. While the exact structure factor is defined from the radial distribution function of the particles, in the case of colloidal images, an estimate for the static structure factor can be obtained by calculating the radial average of the Fourier transform, although at present it is not clear if the coupling of interparticle distance and 
particle size significantly affects the resulting experimental structure factor. This is a topic worth further study.

\section{B.4 Image Analysis in Colloidal Science}

At present, the majority of image analysis methods for colloids have been directed toward digital video microscopy, which are primarily concerned with identifying particle

movements. In a 1995 paper by Crocker and Grier, ${ }^{34}$ digital image processing was used to identify and track particles in videos of colloidal systems in order to estimate diffusion coefficients and pair-wise interaction potentials. In this analysis pipeline, a boxcar average was used to estimate and remove the background, and Gaussian blurring was used to remove digitation noise. Particle positions were estimated by finding local maxima in the pixels and assuming that the brightest pixels were very near particle centers. These particle coordinates were refined by an offset with the weighted centroid of the pixels in the surrounding region. Particles were linked across frames by evaluating the probability of a single Brownian particle diffusing to nearby positions and linking particles with the highest probability of being the same. One major assumption in this analysis was that particles were well separated and had clearly defined boundaries.

Similar techniques were applied to 3D confocal microscopy studies conducted in 2001 by Dinsmore et al. ${ }^{35}$ With well-defined confocal images, they were able to estimate pair distribution functions in binary suspensions and colloidal gels and glasses. Add-ons to the common image analysis software Image ${ }^{36}$ have also been developed to help with analysis of biological cellular and well-defined colloidal systems. One such example is JColloids, which has been successful in micro-rheological studies. ${ }^{37}$ 
Image analysis methods have also been coupled with other experimental data for greater precision. In a study by Milne, Zhao, and Chiu, ${ }^{38}$ TEM was used to calibrate interparticle distances in colloidal monolayers. To process the images, they used filters (unspecified) to clean the images, thresholding to isolate particles, and screening to remove particles lying across the image border or outliers based on brightness, size, and circularity. Center-of-mass of particles were identified and refined using techniques similar to Crocker and Grier. ${ }^{34}$ By assuming a hexagonal close-packed structure, they were able to use this data to reconstruct a model of the system. Similar to the previous studies mentioned, the images they started with had good interparticle definition, which simplified the individual particle detection process considerably.

A completely different approach to bio and colloidal image analysis was built by Berg et al., ${ }^{39}$ who used machine learning to allow the user to "teach" the program what is background and what is an important image feature, and later refined to distinguish between different types of features. This program, Ilastik, ${ }^{40}$ has gained attention from the bioimaging and colloidal imaging community, gaining over 1,000 citations since its v0.5 release in 2011. While this program is extremely flexible and applicable to a wide range of bio/colloidal images, due to statistical uncertainty at particle edges, its use is also limited to images with good boundary definition between particles. 


\section{METHODS}

\section{Overview}

The colloid system analyzed in this experiment was a suspension of silsesquioxane $\left(\mathrm{SiO}_{1.5}\right)$ microspheres stabilized by zirconia $\left(\mathrm{ZrO}_{2}\right)$ held in a solution of $1.5 \mathrm{pH}$ nitric acid $\left(\mathrm{HNO}_{3}\right)$. The colloidal suspensions were separated into $2.5 \mathrm{~mm}$ diameter wells, well mixed on the ISS, and allowed to agglomerate for 8-12 days in microgravity. Images of each well were taken periodically at $2.5 \mathrm{X}$ magnification. To analyze the images and extract agglomeration data, a Python package was developed from standard computer vision, image analysis, and data analysis libraries to automate and standardize the analysis process.

\section{Silsesquioxane Microparticle Synthesis}

Silsesquioxane $\left(\mathrm{SiO}_{1.5}\right)$ microspheres are an organosilicon compound with a cagelike polymeric structure. The silicon center provides rigidity and thermal stability, while the organic exterior enables functionalization. ${ }^{41}$ Benzyl Chloride functionalized Silsesquioxane (BC-SSQ) particles were synthesized using a modified Stöber method. ${ }^{41}$ Rhodamine B functionalization was not used in this experiment but was included in later experiments.

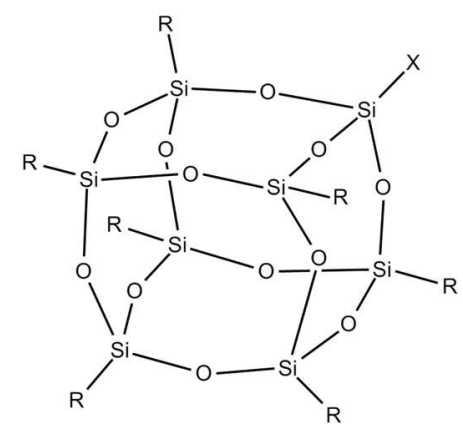

Figure 1: Basic silsesquioxane unit structure 
The silsesquioxane microspheres were advertised as behaving identically to silica, so they were expected to have nearly identical isoelectric points. As a result, the settling experiments were conducted with this assumption. Later testing ${ }^{41}$ found that this was not the case; instead the isoelectric point for this suspension of silsesquioxane was $2.8 \mathrm{pH} .{ }^{42}$

\section{Sample Preparation}

The samples consisted of silsesquioxane microparticles and zirconia nanoparticles at $\mathrm{pH}=1.5$. The silsesquioxane microparticles were $600 \mathrm{~nm}$ in diameter, while the zirconia nanoparticles ranged between $5-15 \mathrm{~nm}$ in diameter, resulting in a size ratio of $\sim 100: 1$ (SiO$1.5: \mathrm{ZrO}_{2}$ ). The volume fraction of $\mathrm{SiO}_{1.5}$ was set to 0.01 . Three concentrations of $\mathrm{ZrO}_{2}$ were tested $-0.01 \%, 0.055 \%$, and $0.1 \%$ by volume. Volume fractions for these three concentrations were $10^{-4}, 10^{-3.26}$, and $10^{-3}$, respectively. The original intent of this experiment was to create a nanoparticle haloing suspension. However, due to the difference between the $\mathrm{pH}$ of this system (1.5) and the true isoelectric point of the system (2.8), it was not a true nanoparticle haloing system, rather a bimodal colloidal system, due to repulsive coulombic interaction between the microspheres.

\section{Microgravity Testing}

Colloidal samples were boarded onto the Cygnus CRS OA-4, also known as the Orb-4, launched on December 6, 2015, destined for the ISS as part of the Nanoparticle Haloing portion of NASA's Advanced Colloids Experiments (ACE-H2). ${ }^{43}$ Agglomeration experiments began on January 4, 2016 and lasted three months. Astronauts mixed the samples in the wells by hand and loaded them into the Light Microscopy Module (LMM), ${ }^{44}$ 
and illuminated them from the side. Due to spatial limitations, side-lighting was required to get enough scattered light to clearly image the particles. This caused a lighting gradient to be present across all experimental images that had to be corrected for in the image analysis. Significant agglomeration had already taken place in the time between when the astronauts had mixed the samples and when the samples had been loaded into the LMM and imaging began. Two potential factors could have contributed to this behavior. First, because the mixing was done by hand, there is not guarantee that the suspensions were well-mixed from the start. Second, the length of time between mixing and imaging was not logged, but is estimated to be between 5-8 hours. Later 2018 and 2021 experiments with similar systems have addressed this issue using sealed capillaries to hold the sample with magnetic micro-stir bars to enable the capture of transient effects immediately after mixing. Results from these experiments will be reported in later papers.

\section{Python - Colloidspy}

Python is an interpreted, open-source, high-level programming language, used in a wide range of applications. In recent years, it has gained increasing use by researchers and data scientists because of its simplicity and short learning curve relative to other text-based programming languages. Thousands of third party, open-source packages are available to add greater functionality to Python environments. ${ }^{45}$ The Python package Colloidspy ${ }^{4}$ was developed specifically for the purpose of analyzing the large quantities of images in this study. Colloidspy makes use of common but powerful computer vision and scientific computing packages, bundling them into functions that are designed to easily analyze microscopic images of colloids. 
To analyze the ACE-H2 images, an on-screen image cropping function was used to select the regions of interest (ROI's) for each agglomeration experiment. Because agglomeration experiments are not agitated once agglomeration begins, the ROI for the first image in the agglomeration sequence was used as the ROI for all images in the sequence. Next, the stack of cropped images was sent through the local thresholding algorithm, based off of scikit-image's gaussian local threshold function, to convert the images to a binary format. According to the documentation, this algorithm computes "a threshold mask image based on local pixel neighborhood. The threshold value is the weighted mean for the local neighborhood of a pixel subtracted by a constant." 28 A lowpixel-value cutoff was added to prevent the interior of large dark portions from showing up as erroneous particles. The optimal block size and offset parameters were determined by visually comparing several binary images to their originals and tuning the parameters until the binary image most closely matched the original, preserving only the particles. An example comparison is shown in Figure 2.
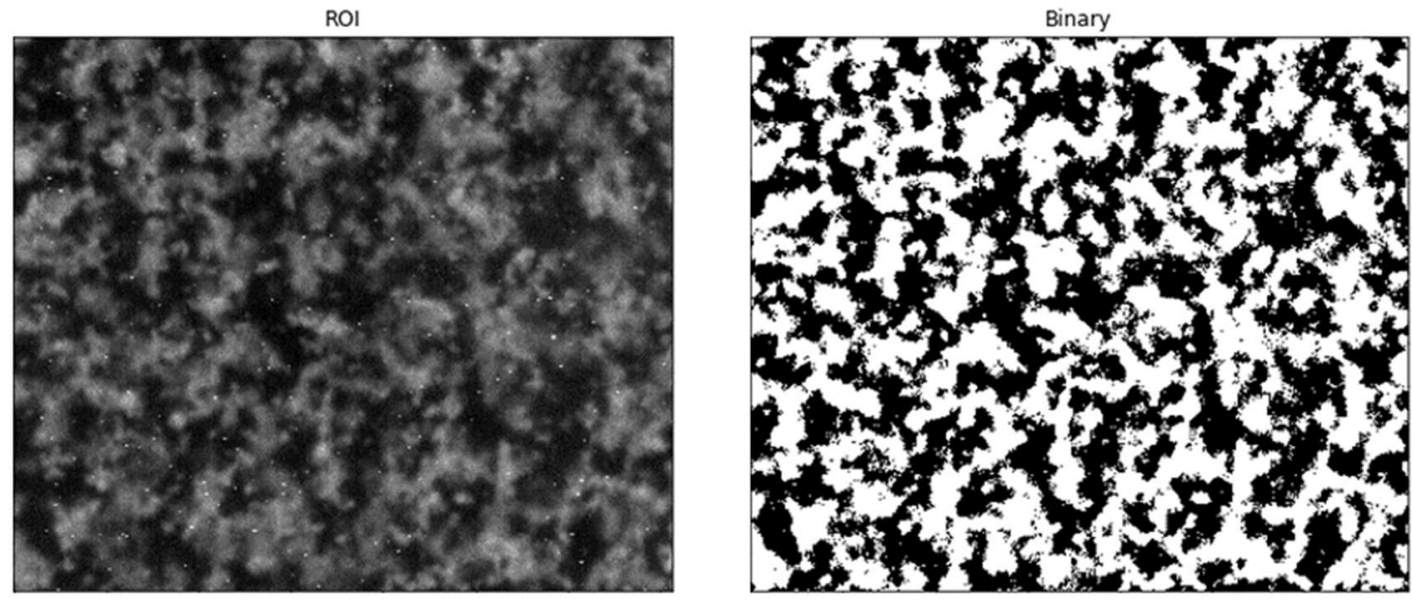

Figure 2: Left - a characteristic sample ROI; Right - the binarized version of the same ROI, where the white portion indicates a cluster 
Next, single-pixel and other gritty imperfections were removed using a binary opening and closing algorithm, which uses successive erosion and dilation to identify and remove features much smaller than the major structuring elements. ${ }^{46}$ A side-by-side example of the raw binary image compared to the cleaned version is shown in Figure 3.
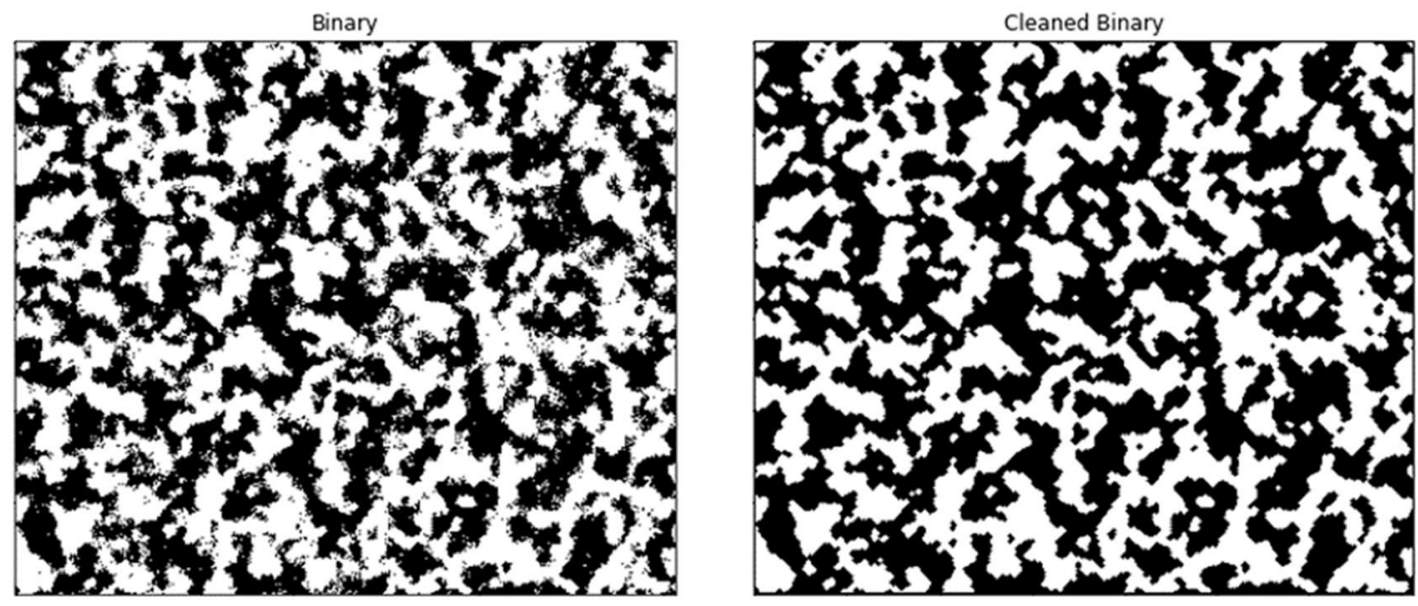

Figure 3: Left - the binary image of an example ROI before cleaning. Right - the same binary image after cleaning

Once the cleaned binary images were generated, the scikit-image watershed algorithm was used to differentiate clusters from each other. This function assigns a number to each cluster and separates clusters that appear to be touching in the binary image but are likely separate clusters. Once the particles were extracted, contour detection from OpenCV was used to characterize each of the clusters. ${ }^{47}$ Cluster area, perimeter, mass center, average convex hull defect length, circularity, and various statistics on those metrics were exported to comma-separated value (csv) files for further analysis. 
As a final note on the development of this image analysis algorithm, simple contour detection without the watershed step was found to result in extremely large clusters, ignoring holes inside clusters, and erroneously considering any clusters touching by only a pixel as part of a single cluster. An example of such a cluster is shown in Figure 4.
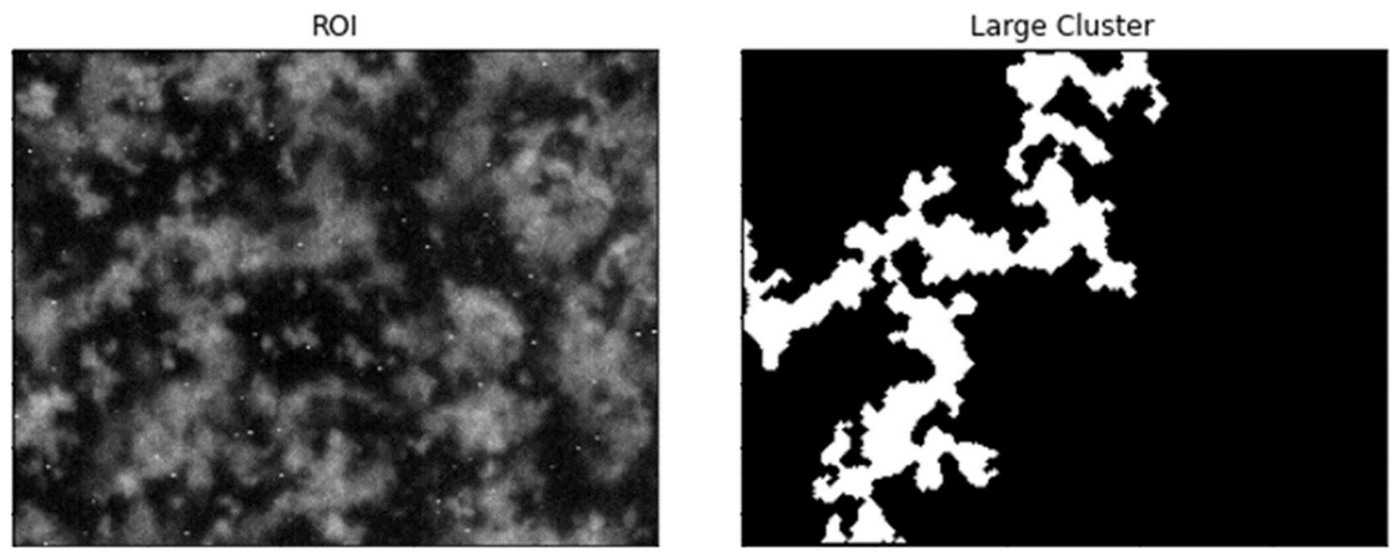

Figure 4: Left - an example ROI; Right - large cluster resulting from contour detection without use of the watershed algorithm

In contrast, the watershed caused some erroneous break-up of large clusters. However, from visual inspection of several test images, the number of clusters that were broken erroneously appeared be a small number compared to the total number of clusters, and were assumed to be a minimal factor in cluster detection. An example of the clusters detected by the watershed algorithm is shown in Figure 5, with cluster boundaries marked in red. Since no more accurate cluster detection algorithm was found upon extensive research on the latest computer vision methods, the total impact of this segmentation error on the results was not quantified. 

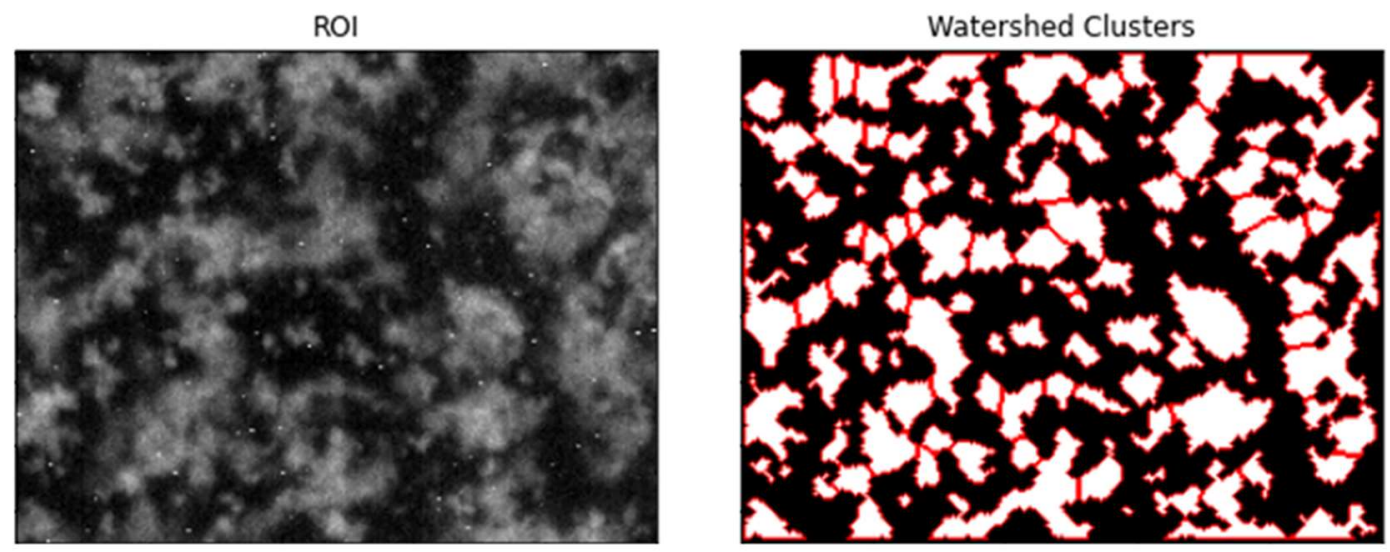

Figure 5: Left - an example ROI; Right - clusters detected by the watershed algorithm are outlined in red, where the white portion is the cluster 


\section{RESULTS AND DISCUSSION}

\section{Cluster Size Analysis}

Average cluster area over time was obtained by averaging the extracted cluster areas from each image and matching them with their corresponding agglomeration times. Shown in Figure 6 is a characteristic example of the average areas for each concentration tested. The $95 \%$ confidence interval for each average area is denoted by the error bars on each datapoint. Regression curves are drawn to guide the eye and do not imply a formal regression proposal. Further study is required to develop an accurate quantitative regression model for these systems.
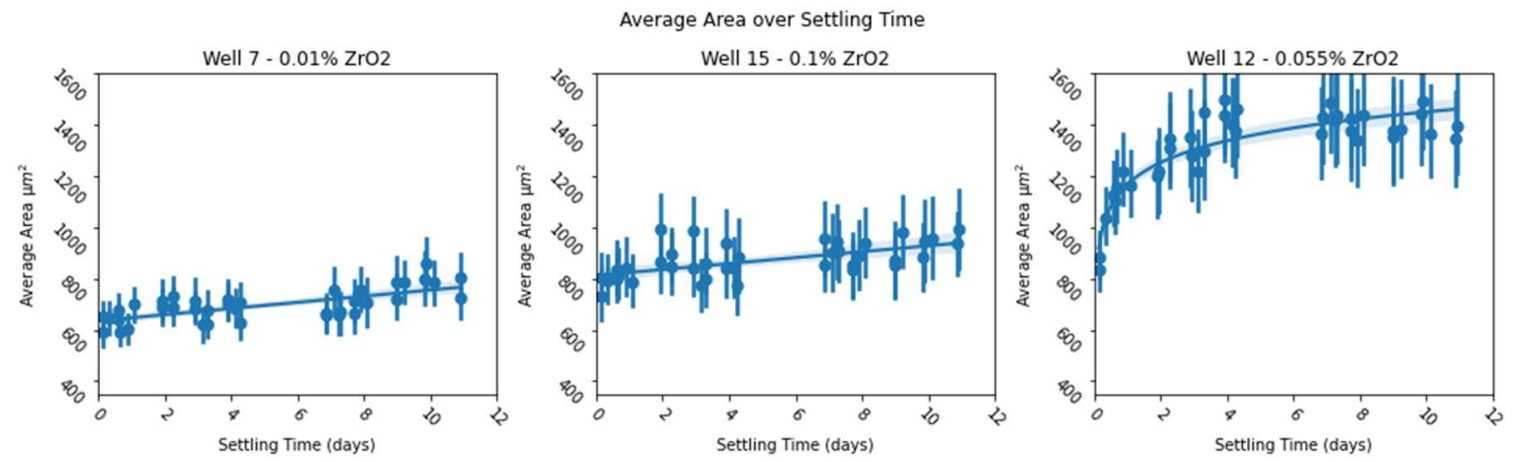

Figure 6: Left - average areas from Well 7, with $0.01 \% \mathrm{ZrO}_{2}$; Middle - average areas

from Well 15, 0.1\% $\mathrm{ZrO}_{2}$; Right - average areas from Well $12-0.055 \% \mathrm{ZrO}_{2}$

In general, the spread of cluster size in each image was larger in regions of greater average cluster size, indicating that the variance in cluster area increases with average particle areas. Consistent with the Central Limit Theorem, ${ }^{48}$ the spread of the average cluster area for all images over time (shown by the shaded $95 \%$ confidence region around 
the regression curves) was smaller than the spread of individual cluster areas for a given image (shown by the error bars on each data point). For the purpose of clarity, later cluster area plots will not include individual error bars but will retain the shaded $95 \%$ confidence region.

A final consideration before examining the remaining cluster size data is the total cluster count. Shown in Figure 7 is the total number of clusters detected for the three characteristic wells analyzed above, plotted over the agglomeration time. Within a closed agglomeration system, the total number of clusters must decrease proportionally with increasing cluster size, as clusters become larger through aggregation of multiple particles. This general behavior is observed as expected: there is a linear decrease in particle count for Wells 7 and 15, similar to the negative of the slope of average cluster area, and a nonlinear decrease in particle count for Well 12, according to the corresponding nonlinear increase in cluster size. Additionally, Figure 8 shows the total spread of cluster count for all wells for all time.
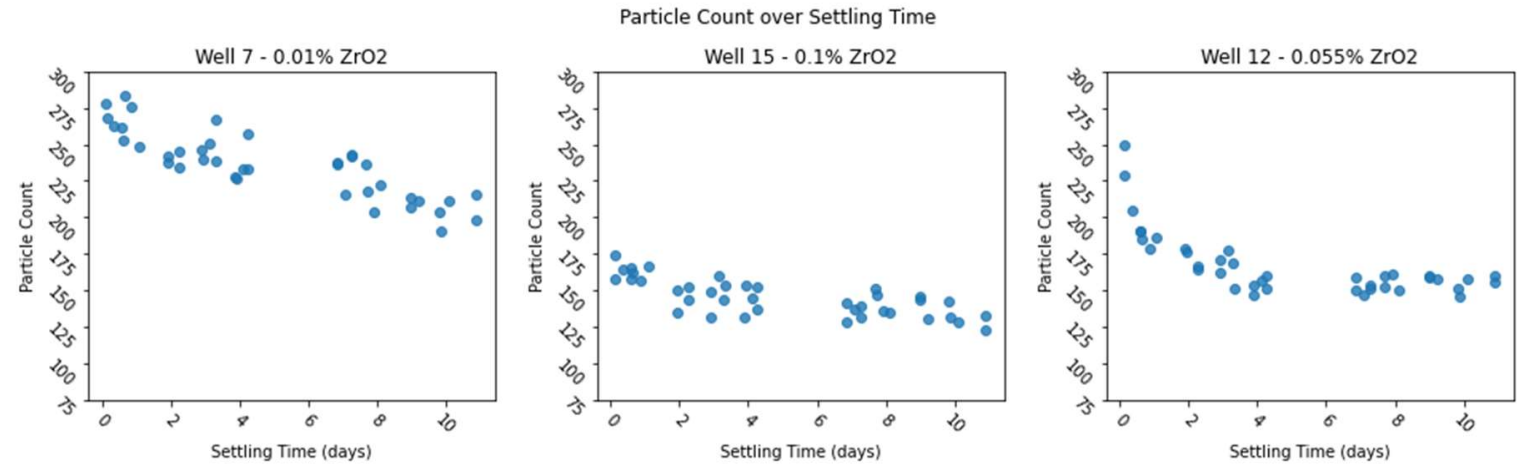

Figure 7: Left - total particle count from Well 7, with $0.01 \% \mathrm{ZrO}_{2}$; Middle - total particle count from Well 15, 0.1\% $\mathrm{ZrO}_{2}$; Right - total particle count from Well $12-0.055 \% \mathrm{ZrO}_{2}$ 


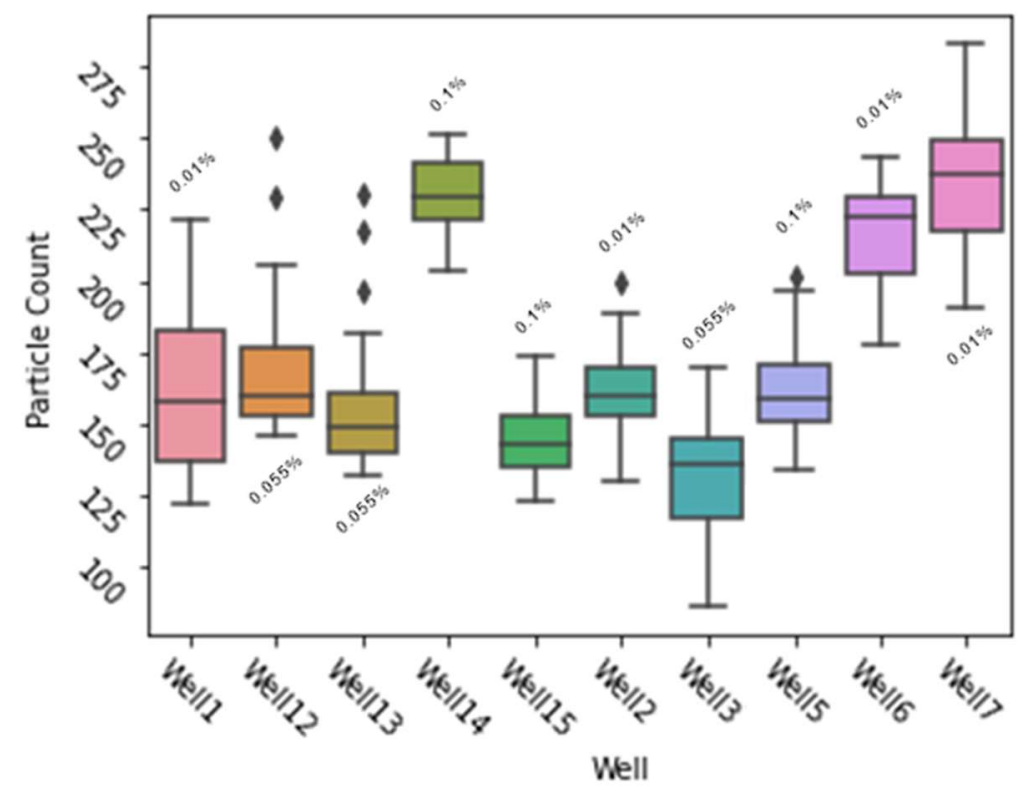

Figure 8: Spread of total particle count over all times for each well

Finally, average cluster area over time was examined, separated by concentration. Shown in Figure 9 is the average cluster area in square microns over agglomeration time in days for all wells with $0.01 \% \mathrm{ZrO}_{2}$ nanoparticle concentration. Again, the regression curves are drawn to guide the eye. 
Average Area over Settling Time - 0.01\% ZrO2
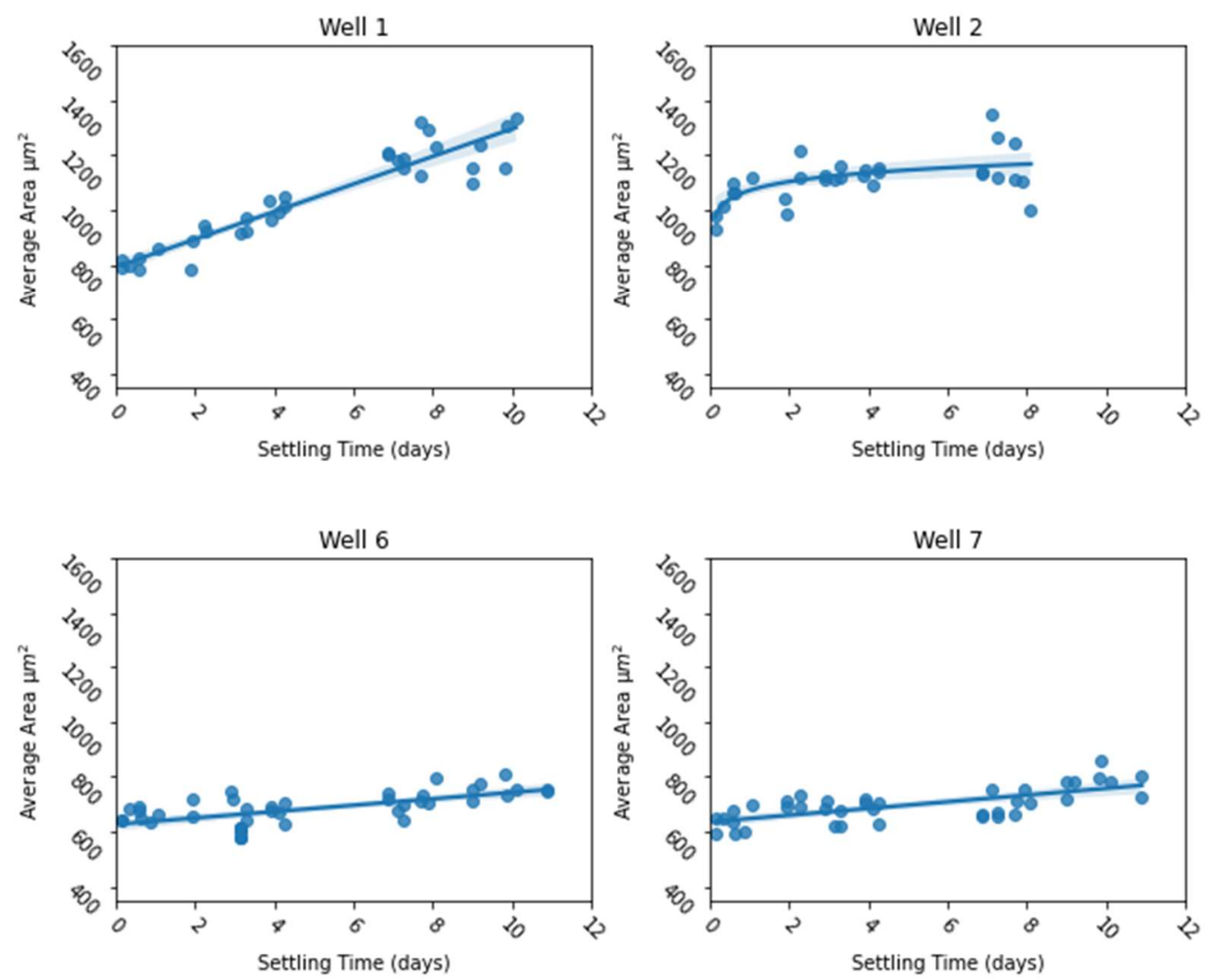

Figure 9: Average cluster area over agglomeration time of all wells with $0.01 \% \mathrm{ZrO}_{2}$ nanoparticles

The changes in average area over time in Wells 2, 6, and 7 appear to be roughly equal, with Well 2 exhibiting the tail-end of a nonlinear cluster growth period in the earliest data points. The smallest average cluster sizes detected in the wells were $\sim 600 \mu \mathrm{m}$, two orders of magnitude higher than the projected area of a single microparticle $\left(1.13 \mu \mathrm{m}^{2}\right)$. Three of the four wells show nearly identical cluster growth rates, which is expected as their systems should be physically identical. The cause of the difference in growth rate observed in Well 1 was not identified. Considering the time gap between the beginning of 
agglomeration after mixing and the first image, the similarity in cluster growth rates, and the magnitude of the starting average cluster sizes, it is highly likely that significant cluster growth occurred before the first images were taken.

Shown in Figure 10 is a similar graphic, but for all wells with $0.055 \% \mathrm{ZrO}_{2}$. Similarly, trendlines are given to guide the eye.
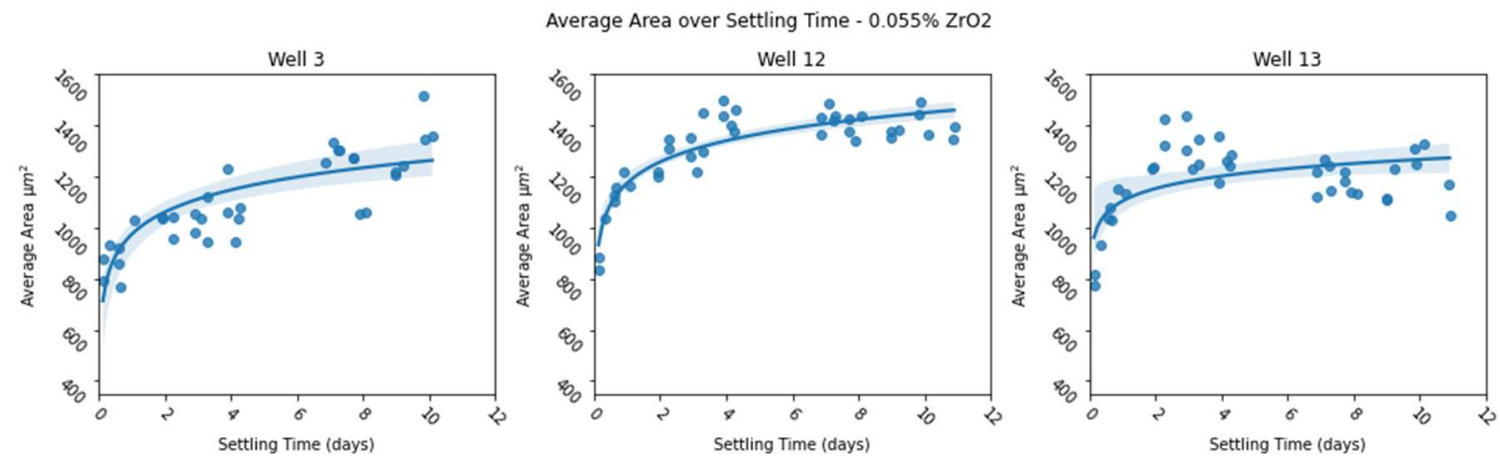

Figure 10: Average cluster area over agglomeration time for all wells with $0.055 \% \mathrm{ZrO}_{2}$ nanoparticles

In these $0.055 \% \mathrm{ZrO}_{2}$ wells, much stronger nonlinear cluster growth appears, consistent with the early trend from Well 2. It is unclear whether this behavior is observed because the first images were taken earlier in the agglomeration period, if the rate of cluster growth was slower so the nonlinear region stretched out longer, or if there simply is more nonlinear behavior at this $\mathrm{ZrO}_{2}$ concentration. Well 4 was omitted from this dataset due to extreme changes in lighting and aggregation of particles onto a stir-bar that was left in the well. Finally, average cluster size over agglomeration time for the $0.1 \% \mathrm{ZrO}_{2}$ nanoparticle concentration wells is shown in Figure 11. 

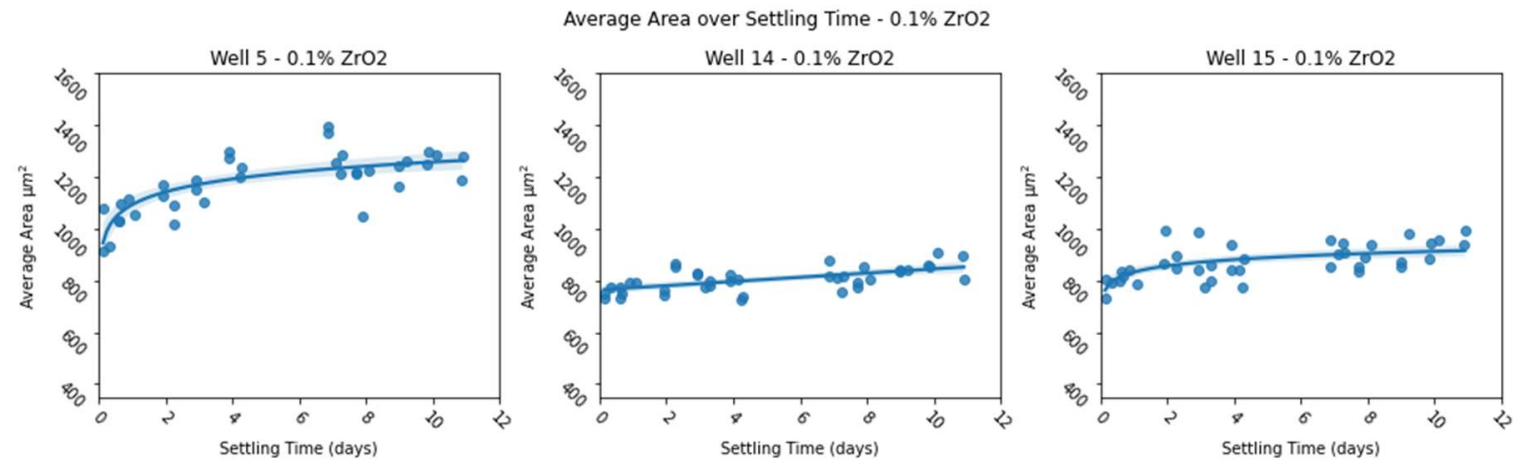

Figure 11: Average cluster area over agglomeration time for all wells with $0.1 \% \mathrm{ZrO}_{2}$ nanoparticles

The first well in the series of $0.1 \% \mathrm{ZrO}_{2}$ wells, Well 5, also shows the tail-end of a nonlinear initial cluster growth period. However, Wells 14 and 15 do not show this phase, instead showing average clusters sizes and slopes consistent with those from Wells 6 and 7 in the $0.01 \% \mathrm{ZrO}_{2}$ group. As before, the low slope in each of these graphs indicates that the majority of cluster growth had already taken place by the time the first image was taken.

\section{Discussion of Results}

The initial expectation for cluster growth for the colloidal systems in this study was to see unbounded, linear cluster growth over time. Calculations done by Walz and Sharma ${ }^{11}$ on charged bimodal colloidal systems based on A-O theory predicted the presence of only attractive interparticle forces in colloidal solutions with concentrations less than about $0.5 \%$ of the nanoparticles for a 1000:1 size ratio. In the case of this experiment, the nanoparticle concentrations tested were $0.01 \%, 0.055 \%$, and $0.1 \%$ at size 
ratio $\sim 100: 1$. While the size asymmetries in their system verses the systems tested in this study are different, the drastic difference in agglomeration behavior was not expected.

Theory developed by Chu et al. ${ }^{12}$ predicts that decreasing size ratio should strongly decrease the repulsive peak just after the attractive potential well, and increasing secondary phase concentration should strongly increase the depth of the potential well while moderately increasing the height of the energy barrier. While these two factors counterbalance each other, the strong action on the energy barrier by decreasing size asymmetry should increase colloidal aggregation. Similarly, increasing the secondary phase concentration should increase aggregation rate. In this study, secondary phase concentrations were all much lower than in Walz and Sharma's system, and size asymmetry was also lower. Therefore, by the theoretical trends from Walz \& Sharma and Chu et al., the colloidal systems in this study should have shown linear aggregation rates, and the rates should increase with increasing $\mathrm{ZrO}_{2}$ concentration.

Observing only the concentration endpoints, colloidal aggregation rates in the $0.01 \%$ and $0.1 \% \mathrm{ZrO}_{2}$ wells are very similar, with the exception of Well 1, contrary to the expectation of increasing aggregation rate. The cause for the high aggregation rate in Well 1 compared to all other wells is unknown. If Well 1's data was not compromised or an anomaly, its aggregation rate still contradicts the expectations from theory, along with the other wells. Including or rejecting the dataset would not change the final conclusions drawn, so further investigation was not deemed necessary at this point in the study.

The intermediate concentration level, $0.055 \% \mathrm{ZrO}_{2}$, showed very unexpected nonlinear aggregation behavior. All three wells started at low relative cluster sizes, increased very quickly, and then mostly stabilized. No reference has been made in theory 
to a critical cluster size, however some studies ${ }^{13,14}$ have found that a high concentration of the secondary phase can cause quasi-stabilization through inhibition of movement. It may be possible that in the absence of gravity, the thermal energy of large clusters is not enough to sustain constant aggregation rates as the distance between clusters increases, and similar behavior exists as with high secondary phase concentration. In gravity, the space between clusters may be decreased through buoyancy forces, allowing continued aggregation where our microgravity system may be limited by transport mechanisms due to the lack of buoyancy forces. Further study into this phenomenon is highly encouraged. In particular, a study using bursts of frequent imaging coupled with particle tracking methods such as Trackpy $^{3}$ could be used to directly study the changes in mean free path for clusters in similar microgravity suspensions. By this hypothesis, the mean free path should decrease as cluster size increases, and particle movement should be slow enough that clusters do not significantly interact with other clusters on their random-walks after the initial rapid growth phase.

The remaining unanswered question is why do the $0.055 \% \mathrm{ZrO}_{2}$ wells show such strong nonlinear aggregation rates, while the other two concentrations do not? The average aggregations rates in all concentrations, even in the region just past the nonlinear portion in the intermediate concentration wells, are strikingly similar. Because the mixing of each well was done manually by an astronaut on the ISS just before the start of agglomeration, as many as $5-8 \mathrm{hrs}$ had passed between the initial mixing of the wells and when first agglomeration images taken. Therefore, it is certain that significant aggregation had already occurred by the time the first images were taken. If all wells actually exhibited the characteristic nonlinear behavior seen in the intermediate concentration wells $(3,12,13)$, 
the initial rapid aggregation phase could easily have been missed and the recorded data could have been obtained only from the slow aggregation or quasi-stabilized time frames. This hypothesis is supported by the presence of the tail-ends of nonlinear aggregation shown at the beginnings of Wells 2 and 5. Later experiments performed on the ISS in 2018 and 2021 have addressed this issue by using a magnetic micro-stir bar to minimize the interim time between mixing and imaging to capture the transient effects. Analysis of this later data was beyond the scope of this thesis.

\section{Sources of Error}

As outlined in the Methods section, the Python package that was used to analyze the images in this study uses a watershed function to identify and separate clusters from each other, even if they appear to be touching in the image. Visual inspection comparing raw images to their corresponding binary images showing only the clusters found this method to be largely accurate - the vast majority of the darker regions between touching clusters in the raw images were correctly identified and separated by the watershed. However, some of the larger clusters were falsely split by the watershed function. As a result, there is a possibility that some larger clusters are not accurately represented in the cluster data. While this could have been avoided by setting a larger minimum distance between cluster centers, this would have caused many smaller clusters to be completely filtered out. Further study comparing the impact of minimum cluster distance on final data is required. Due to the unavailability of more robust particle detection algorithms, more accurate comparison data will be extremely difficult to obtain. 
A similar source of error is nonlinearity in the expected cluster growth. Because this study measured the projected cluster size onto a plane, while the agglomeration rate may be linear, the projected area onto the plane is nonlinear. Eggersdorfer et al. ${ }^{49}$ studied the structure of agglomerates, showing that agglomerates grown by particle collision do exhibit power law scaling, as shown in Equation 4. The power law prefactor and exponent must be determined experimentally by methods such as nitrogen adsorption or small angle Xray scattering. ${ }^{49}$

$$
n_{p}=k_{a}\left(\frac{a_{a}}{a_{p}}\right)^{D_{\alpha}}
$$

Where: $\mathrm{n}_{\mathrm{p}}=$ number of particles

$a_{p}=$ projected area of a single particle

$\mathrm{a}_{\mathrm{a}}=$ projected area of the agglomerate

$\mathrm{k}_{\mathrm{a}}, \mathrm{D}_{\alpha}=$ power law prefactor and exponent, respectively

Using the projected area of individual silsesquioxane particles, a prefactor of 1.0, and a generous exponential value of 2.0 , chosen from upper range of exponentials reported in the study, Figure 12 was constructed to assess the impact of this nonlinearity. 


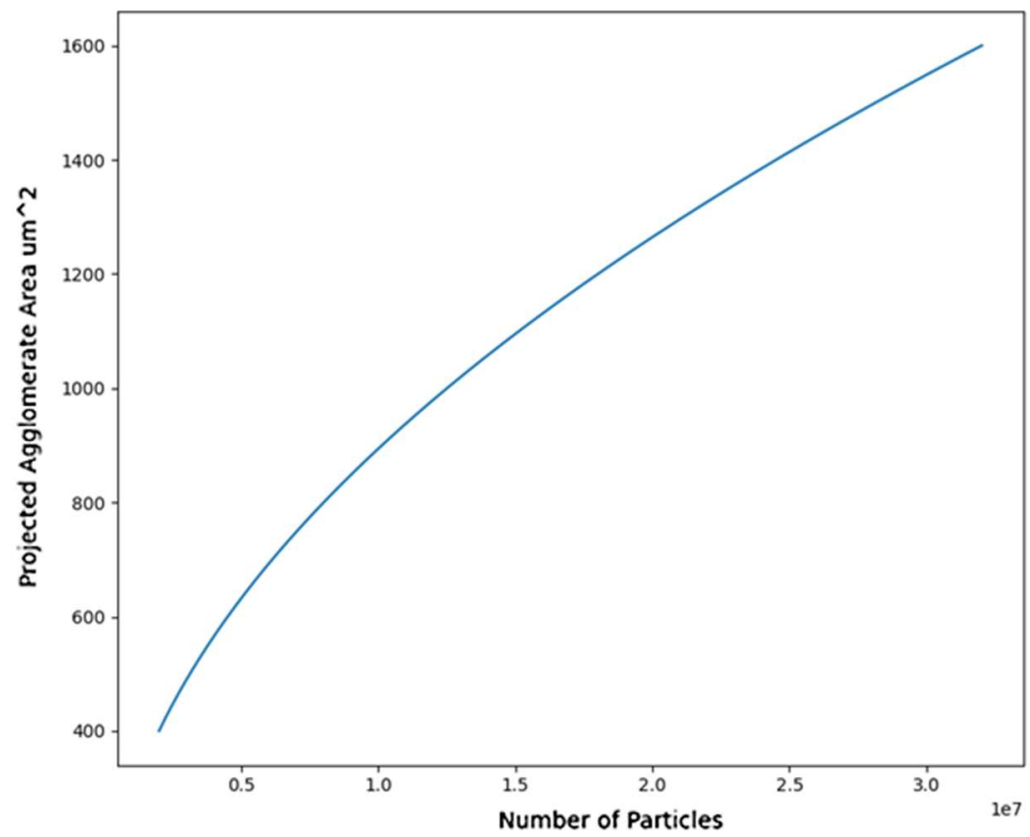

Figure 12: Projected agglomerate area over number of particles

The nonlinearity in agglomerate area by the number of particles is vastly less than the nonlinearity observed in the agglomerate area from the experimental images over time. The observed nonlinearity only appeared at large exponents $>5$, far outside of the bounds of exponents expected for typical colloidal suspensions. If there is additional nonlinearity between number of particles added to the agglomeration over time, rate of particle addition is likely to increase at larger agglomerate sizes as more surface area is exposed to the bulk to attract smaller particles. In this case, the nonlinearity between number of particles over time would counteract the nonlinearity shown in Figure 12, causing the system to be more linear.

Ilastik $^{39}$, a Python-based tool that utilizes machine learning to detect and analyze particles, was tested as an alternate image analysis method. The program was trained by 
tracing out sections of the background and filling in clusters by hand. A machine learning algorithm was applied to that dataset to identify trends in image characteristics using regression analysis, and gave predictions on cluster positions and boundaries in the remaining images. On account of several factors - unclear cluster boundaries, irregular cluster morphology, uneven lighting - the predictions of the algorithm yielded extremely large clusters with boundaries that resembled clouds more than discrete aggregates. Further training resulted in only nominal improvements, so the method was abandoned.

The most significant source of error in this study is the amount of time between the mixing of the samples and the start of imaging. Judging by the slopes of the trendlines for cluster size over time, as well as the large magnitude of starting cluster sizes compared to individual microparticle sizes, significant agglomeration had already taken place by the time the first images had been taken several hours later. The exact amount of time between mixing and start of imaging is unknown, so it is impossible to know the agglomeration rate within that time frame with any certainty. As mentioned previously, this issue was addressed in later experiments, which are outside the scope of this thesis. 


\section{CONCLUSIONS}

In this study, suspensions of $1 \mathrm{vol} \%$ silsesquioxane microparticles and $0.01,0.055$, and 0.1 vol\% zirconia nanoparticles were allowed to agglomerate in microgravity for 8-12 days, imaged periodically at $2.5 \mathrm{X}$ to study their agglomeration behavior. Because of the high density of clusters, the lack of clear boundaries between particles within clusters, and lighting gradients, existing colloidal analysis methods were incapable of providing accurate and meaningful results. To address this, the Python package Colloidspy ${ }^{4}$ was developed using robust computer vision and data analysis packages to segment clusters as accurately as possible and extract cluster data and statistics. Previous analyses on this system had been conducted using ImageJ, which yielded reasonable results, but lacked the flexibility and automation capability required to standardize the analysis pipeline and still return accurate results, in addition to requiring the use of other programs to analyze the data it extracts.

Contrary to existing theory which predicts linear cluster growth within systems and increasing agglomeration rate with increasing nanoparticle concentration, nonlinear cluster growth was observed, starting with rapid agglomeration, increasing to a plateau indicating quasi-stabilization. This behavior is more consistent with transport limited agglomeration than the traditionally proposed thermodynamic mechanisms. Further study into the effect

of transport mechanisms such as lack of buoyancy on the agglomeration of thermodynamically unstable systems in microgravity should be conducted. One possible route is to use particle tracking to study the mean free path of clusters and assess the influence of transport on aggregation in microgravity. 


\section{REFERENCES}

1. Mourdikoudis, S.; Pallares, R. M.; Thanh, N. T. K., Characterization techniques for nanoparticles: comparison and complementarity upon studying nanoparticle properties. Nanoscale 2018, 10 (27), 12871-12934.

2. Ohshima, H.; Healy, T. W.; White, L. R.; O'Brien, R. W., Sedimentation velocity and potential in a dilute suspension of charged spherical colloidal particles. Journal of the Chemical Society, Faraday Transactions 2: Molecular and Chemical Physics 1984, 80 (10), 1299-1317.

3. W Perry; François Boulogne; Prashant Sinha; pfigliozzi; Nicolas Bruot; Leonardo Uieda; Jan Katins; Hadrien Mary; Aron Ahmadia., D. A. C. v. d. W. N. K. T. A. C. D. W. R. V. C. R. T. L. G. K. R. a. z. R. Soft-matter/trackpy, Trackpy V0.4.2; Zenodo, 2019.

4. Cecil, A. Colloidspy, 2021.2; Github: 2021.

5. Emin, S.; Singh, S. P.; Han, L.; Satoh, N.; Islam, A., Colloidal quantum dot solar cells. Solar Energy 2011, 85 (6), 1264-1282.

6. Zhu, C.; Pascall, A. J.; Dudukovic, N.; Worsley, M. A.; Kuntz, J. D.; Duoss, E. B.; Spadaccini, C. M., Colloidal Materials for 3D Printing. Annual Review of Chemical and Biomolecular Engineering 2019, 10 (1), 17-42.

7. Shibayama, M.; Li, X.; Sakai, T., Gels: From Soft Matter to BioMatter. Industrial \& Engineering Chemistry Research 2018, 57 (4), 1121-1128.

8. Selmi, F., Intorno all'azione dell'iodio sopra il clorido di mercurio: memoria prima di Francesco Selmi. Milano, 1845.

9. Einstein, A., Über die von der molekularkinetischen Theorie der Wärme geforderte Bewegung von in ruhenden Flüssigkeiten suspendierten Teilchen. Annalen der Physik 1905, $322(8), 549-560$.

10. Asakura, S.; Oosawa, F., Interaction between particles suspended in solutions of macromolecules. Journal of Polymer Science 1958, 33 (126), 183-192.

11. Walz, J. Y.; Sharma, A., Effect of Long Range Interactions on the Depletion Force between Colloidal Particles. Journal of Colloid and Interface Science 1994, 168 (2), 485496.

12. Chu, X. L.; Nikolov, A. D.; Wasan, D. T., Effects of Particle Size and Polydispersity on the Depletion and Structural Forces in Colloidal Dispersions. Langmuir 1996, 12 (21), 5004-5010.

13. Liu, J.; Shih, W. Y.; Kikuchi, R.; Aksay, I. A., Clustering of binary colloidal suspensions: Theory. Journal of Colloid and Interface Science 1991, 142 (2), 369-377.

14. Yasrebi, M.; Shih, W. Y.; Aksay, I. A., Clustering of binary colloidal suspensions: Experiment. Journal of Colloid and Interface Science 1991, 142 (2), 357-368.

15. Mo, S.; Shao, X.; Chen, Y.; Cheng, Z., Increasing entropy for colloidal stabilization. Scientific Reports 2016, 6 (1), 36836.

16. Tohver, V.; Chan, A.; Sakurada, O.; Lewis, J. A., Nanoparticle Engineering of Complex Fluid Behavior. Langmuir 2001, 17 (26), 8414-8421.

17. He, Q. Investigation of stabilization mechanisms for colloidal suspension using nanoparticles. University of Louisville, Electronic Theses and Dissertations, 2014.

18. Chan, A. T.; Lewis, J. A., Electrostatically Tuned Interactions in Silica Microsphere-Polystyrene Nanoparticle Mixtures. Langmuir 2005, 21 (19), 8576-8579. 
19. Chan, A. T., Size Ratio Effects on Interparticle Interactions and Phase Behavior of Microsphere-Nanoparticle Mixtures. Langmuir 2008, 24 (20), 11399-11405.

20. Weight, B. M.; Denton, A. R., Structure and stability of charged colloid-nanoparticle mixtures. The Journal of Chemical Physics 2018, 148 (11), 114904.

21. Jung, S.-K., A Review of Image Analysis in Biochemical Engineering. Biotechnology and Bioprocess Engineering 2019, 24 (1), 65-75.

22. Greensted, A. Otsu Thresholding.

http://www.labbookpages.co.uk/software/imgProc/otsuThreshold.html (accessed

3/2/2021).

23. Local Adaptive Thresholding. In Encyclopedia of Biometrics, Li, S. Z.; Jain, A., Eds. Springer US: Boston, MA, 2009; pp 939-939.

24. Dnyandeo, S. V. N., R.S., A Review of Adaptive Thresholding Techniques for Vehicle Number Plate Recognition. International Journal of Advanced Research in Computer and Communication Engineering (IJARCCE) 2016, 5 (4), 944-946.

25. Sankur, B. L., Survey over image thresholding techniques and quantitative performance evaluation. Journal of Electronic Imaging 2004, 13 (1), 146.

26. Jain, R. K., R.; Schunck, B., Image Filtering. In Machine Vision, McGraw-Hill, Inc.: 1995; pp 112-137.

27. Fisher, R. P., S.; Walker, A.; Wolfart, E. Gaussian Smoothing. https://homepages.inf.ed.ac.uk/rbf/HIPR2/gsmooth.htm.

28. Van Der Walt, S.; Schönberger, J. L.; Nunez-Iglesias, J.; Boulogne, F.; Warner, J. D.; Yager, N.; Gouillart, E.; Yu, T., scikit-image: image processing in Python. PeerJ 2014, 2 , e453.

29. Fisher, R. P., S.; Walker, A.; Wolfart, E. Sobel Edge Detector. https://homepages.inf.ed.ac.uk/rbf/HIPR2/sobel.htm (accessed 3/3/2021).

30. Keim, R. What Is the Fourier Transform All About Circuits [Online], 2020. https://www.allaboutcircuits.com/technical-articles/what-is-the-fourier-transform/

(accessed 3/3/2021).

31. Weisstein, E. W. Fourier Transform Wolfram MathWorld--A Wolfram Web Resource [Online], 2021. https://mathworld.wolfram.com/FourierTransform.html (accessed $3 / 3 / 2021)$.

32. Fisher, R. P., S.; Walker, A.; Wolfart, E. Fourier Transform. https://homepages.inf.ed.ac.uk/rbf/HIPR2/fourier.htm.

33. Sjögren, L., Chapter 9 - Colloidal Suspensions. University of Gothenburg: pp 101-130.

34. Crocker, J. C. G., D.G., Methods of Digital Video Microscopy for Colloidal Studies. Journal of Colloid and Interface Science 1996, 179, 298-310.

35. Dinsmore, A. D.; Weeks, E. R.; Prasad, V.; Levitt, A. C.; Weitz, D. A., Threedimensional confocal microscopy of colloids. Applied Optics 2001, 40 (24), 4152.

36. Rueden, C. T.; Schindelin, J.; Hiner, M. C.; Dezonia, B. E.; Walter, A. E.; Arena, E. T.; Eliceiri, K. W., ImageJ2: ImageJ for the next generation of scientific image data. BMC Bioinformatics 2017, 18 (1).

37. Domínguez-García, P.; Pancorbo, M.; Ortega, F.; Rubio, M. A., JColloids: Image analysis for video-microscopy studies of colloidal suspensions. Computer Physics Communications 2018, 231, 243-244. 
38. Milne, G.; Zhao, Y.; Chiu, D. T., High-Precision Measurement and Analysis of Colloidal Monolayers. Analytical Chemistry 2010, 82 (9), 3943-3949.

39. Berg, S.; Kutra, D.; Kroeger, T.; Straehle, C. N.; Kausler, B. X.; Haubold, C.; Schiegg, M.; Ales, J.; Beier, T.; Rudy, M.; Eren, K.; Cervantes, J. I.; Xu, B.; Beuttenmueller, F.; Wolny, A.; Zhang, C.; Koethe, U.; Hamprecht, F. A.; Kreshuk, A., ilastik: interactive machine learning for (bio)image analysis. Nature Methods 2019, 16 (12), 1226-1232.

40. Berg, e. a. Ilastik, 1.3.3; 2020.

41. Moradi, M.; Woods, B. M.; Rathnayake, H.; Williams, S. J.; Willing, G. A., Effect of functionalization on the properties of silsesquioxane: a comparison to silica. Colloid and Polymer Science 2019, 297 (5), 697-704.

42. Hawtrey, L.; Hawtrey, L. T.; MICROGRAVITY, H. I. In Stabilization of bimodal colloidal systems via nanoparticle haloing in microgravity, 2020.

43. Graham, S. R., Timothy Advanced Colloids Experiments (ACE). https://www1.grc.nasa.gov/space/iss-research/iss-fcf/fir/lmm/ace/ (accessed 3/6/2021).

44. NASA LMM | Glenn Space Center | NASA. (accessed 1/11/2021).

45. Python, S. F. Python, 3.8; 2021.

46. Virtanen, P.; Gommers, R.; Oliphant, T. E.; Haberland, M.; Reddy, T.; Cournapeau,

D.; Burovski, E.; Peterson, P.; Weckesser, W.; Bright, J.; Van Der Walt, S. J.; Brett, M.; Wilson, J.; Millman, K. J.; Mayorov, N.; Nelson, A. R. J.; Jones, E.; Kern, R.; Larson, E.; Carey, C. J.; Polat, I.; Feng, Y.; Moore, E. W.; Vanderplas, J.; Laxalde, D.; Perktold, J.; Cimrman, R.; Henriksen, I.; Quintero, E. A.; Harris, C. R.; Archibald, A. M.; Ribeiro, A. H.; Pedregosa, F.; Van Mulbregt, P., SciPy 1.0: fundamental algorithms for scientific computing in Python. Nature Methods 2020, 17 (3), 261-272.

47. Bradski, G., The OpenCV Library. Dr. Dobb's Journal of Software Tools 2000.

48. Weisstein, E. W. Central Limit Theorem Wolfram MathWorld--A Wolfram Web Resource [Online], 2021. https://mathworld.wolfram.com/CentralLimitTheorem.html (accessed 3/3/2021).

49. Eggersdorfer, M. L.; Pratsinis, S. E., The Structure of Agglomerates Consisting of Polydisperse Particles. Aerosol Science and Technology 2012, 46 (3), 347-353. 